\title{
Controlling walking Period of a Pneumatic Muscle Walker
}

\author{
Takashi TAKUMA ${ }^{1}$, Koh HOSODA ${ }^{12}$, Masaki OGINO ${ }^{1}$, Minoru ASADA ${ }^{12}$ \\ 1 Dept. of Adaptive Machine Systems, Graduate School of Engineering, \\ 2 HANDAI Frontier Research Center \\ \{takuma, ogino\}@er.ams.eng.osaka-u.ac.jp \\ \{hosoda, asada\}@ams.eng.osaka-u.ac.jp
}

Summary. A passive dynamic walker can walk down a shallow slope without any actuation. If the dynamics of the biped is effectively utilized to realize passive walking, the robot walks with energy-less locomotion. An antagonistic pair of pneumatic actuators is one candidate to realize such quasi-passive walking using both passive and active motions like some animals do. This paper presents a simple control of to enlarge the stability of biped walking utilizing passive dynamics. First, we introduce the design of the biped robot used for experiments with antagonistic pairs of pneumatic actuators. Then, we propose to regulate opening duration of the valves based on the observed walking cycle. Finally, experimental results are shown to demonstrate the effectiveness of the proposed control scheme.

\section{Introduction}

A passive dynamic walker can walk down a shallow slope without any actuation[1], and is regarded to be one key technology for realizing human-like biped walking not only on slope but on various terrain. If the dynamics of the biped is effectively utilized, we can decrease the effort to design the desired trajectories for joints, eliminate several actuators, and realize less energy consumption with biped walking. To realize such quasi-passive dynamic walkers, the actuators should realize both active and passive motions. Although several researchers adopted electrical motors with reduction gears that can provide large torque [4,5], the back-drivability cannot be preserved because of its friction, and therefore, it is difficult to swing the leg in a passive manner. Sugimoto and Osuka adopted direct-drive motors which made the weight of the robot heavy [3].

An antagonistic pair of pneumatic actuators should be one candidate to realize both passive and active motions like some animals do. Wisse et al. built a biped robot with such pairs of pneumatic actuators, and realized less energy consumption biped walking by utilizing the passive dynamics [2]. They adjusted opening duration of air-supply valves by a trial and error manner. The stability of the walking almost depended on the robot dynamics since the duration is fixed, and as a result, the negotiable terrain is relatively limited. 
If the pairs of actuators are controlled to track a given trajectory, they suffer from their friction and hysteresis. Although several researchers proposed control schemes to compensate such non-linearity by utilizing the dynamic models $[7,8]$, or by using fast switching on/off valves [9], the performance was so far not satisfactory. If the robot dynamics is properly utilized, the trajectory tracking control is not necessary for walking stability. In this paper, a simple control of the duration is proposed to enlarge the stability of the biped walking utilizing passive dynamics. First, we introduce the design of the biped robot used for experiments with antagonistic pairs of pneumatic actuators. Then, we propose to regulate opening duration of the valves based on the observed walking cycle. Finally, the experimental results are shown to demonstrate the effectiveness of the proposed control scheme.

\section{Development of a biped walker with antagonistic pairs of pneumatic actuators}

We have developed a biped robot driven by antagonistic pairs of pneumatic actuators shown in Fig.1. We adopted McKibben artificial muscles [6] made by HITACHI Medical Corporation [10]. Its length and radius are $0.2[\mathrm{~m}]$ and $0.020[\mathrm{~m}]$ (when it contracts), respectively. It generates approx. $800[\mathrm{~N}]$ when the pressure in the inner tube is $0.7[\mathrm{MPa}]$. Fig. 1 shows an overview of a control system for one joint driven by an antagonistic pair of actuators. Each actuator has supply and exhaust valves (SMC Corporation[11]) that are controlled by a D/A converter through an amplifier. Pressure inside the actuator is measured by a pressure sensor and the sensory datas are obtained through an A/D converter.

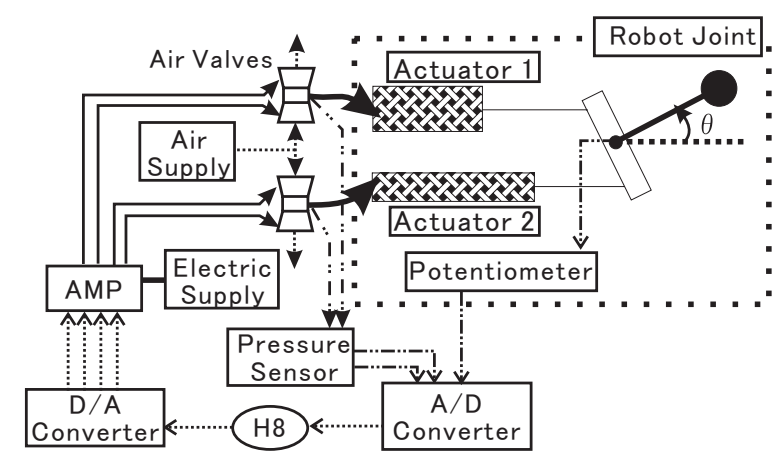

Fig. 1. Overview of the system

Fig. 2 shows the structure of the developed planar walking robot. The height, width, and weight of the robot are $0.750[\mathrm{~m}], 0.350[\mathrm{~m}]$, and $5[\mathrm{~kg}]$, respectively. It has four legs: two connected pairs of legs (outer and inner). Lengths of thigh and shank are $0.3[\mathrm{~m}]$ and $0.35[\mathrm{~m}]$, whose weights are $2.16[\mathrm{~kg}]$ and $0.48[\mathrm{~kg}]$, respectively. It has a micro computer(H8/3067) with D/A and A/D converters (i). As it is designed to be self-sufficient, it has two $\mathrm{CO}_{2}$ bottles on it as air sources each of which weighs $0.7[\mathrm{~kg}](\mathrm{ii})$. The pressure is $1.2[\mathrm{MPa}]$ and is regulated by a pressure regulator into 
$0.7[\mathrm{MPa}]$. It has round-shape soles whose curvature and length are $0.125[\mathrm{~m}]$ and $0.16[\mathrm{~m}]$, respectively, determined by trial and error (iv). The sole has an ON/OFF switch that detects collision with the ground.

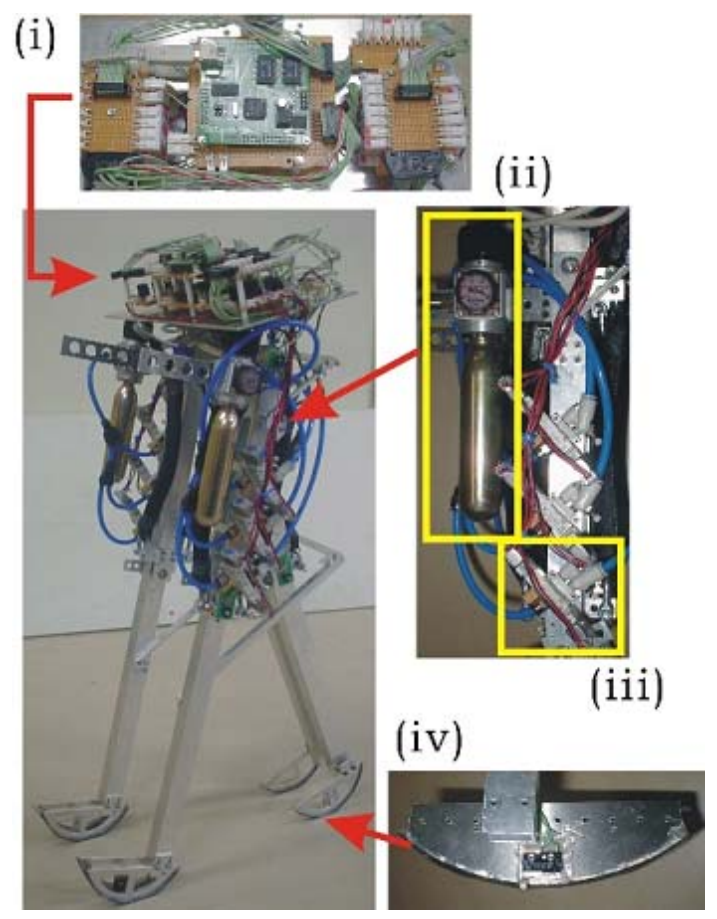

Fig. 2. Real biped walker with McKibben muscle actuators.

\section{Regulation of valve-opening duration for stabilizing biped walking}

\subsection{Relation between robot design and control}

If we can apply model-based control, we can ensure strict stability of the robot. However, we cannot apply such model-based control since it is hard to model the complicated interaction between the robot and the terrain. If the biped is properly designed to realize passive dynamic walking, on the other hand, it is expected that it can negotiate various terrains with little control. Simple control should be, then, enough for increasing the walking stability.

In this paper, we focus on the relation between the walking cycle and the opening duration of the valves and propose to regulate opening duration of the valves based on the observed walking cycle. Since the biped is properly designed to realize quasipassive dynamic walking, we can let the robot walk even with small perturbation 
in the control, that is, perturbation in the duration. We can plot, then, the relation between the duration and the walking cycle that is observed by the touch sensors. Although the strict stability is not proved, we may find an appropriate controller.

If we can find a weak-linear relation between the duration and the walking cycle like depicted in Fig.3, we can apply feedback control based on the relation, which may increase the stability of the biped walking utilizing passive dynamics:

$$
S(k)=S(k-1)-K\left(T(k-1)-T_{d}\right),
$$

where $S(k), T(k), T_{d}, K$ are the opening duration of the valve, the walking cycle at the $k$-th step, the desired walking cycle, and the feedback gain that is determined by the weak-linear relation, respectively.

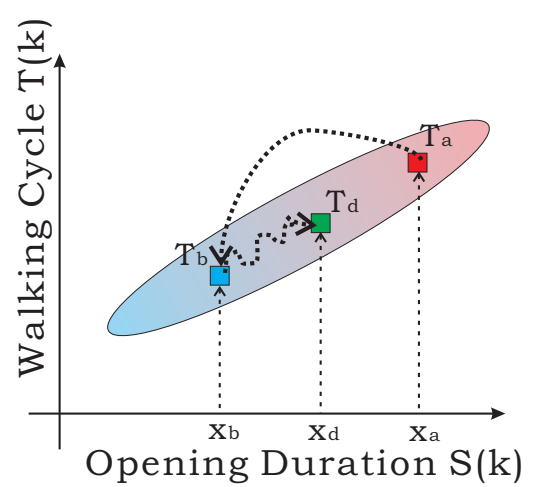

Fig. 3. Correlation between walking cycle and manipulated variable. Following the control law (1), the duration of opening valves is set $x_{b}$ when the walking cycle is slower than desired cycle $T_{d}$. It is expected that the walking cycle changes faster from $T_{a}$ to $T_{b}$, and then converges to desired cycle $T_{d}$.

\subsection{Stabilizing walking by regulating opening duration of valves}

We operate the valves to generate a walking pattern shown in Fig.4. The sequence is:

(i) at the beginning of $\mathrm{k}$-th step, all valves are closed until $T_{0}[\mathrm{~s}]$ (a fixed value determined by trial and error),

(ii) At $T_{0}[\mathrm{~s}]$, the supply valve of the hip flexor and exhaust valve of the hip extensor are opened for $S(k)[\mathrm{s}]$. During this period, the knee of the free leg first bends, and then, stretch to be ready for the coming impact.

(iii) all valves are closed so that the robot keeps the posture until the free leg touches the ground.

In the operations (i) and (iii), the collision with the ground is detected by the switch on the sole shown in Fig.2(iv). Operations (i)-(iii) correspond to items (i)-(iii) in Fig.4, respectively. Let $T(k)$ be a walking cycle from the heel strike to the other heel strike at $k$-th step. 


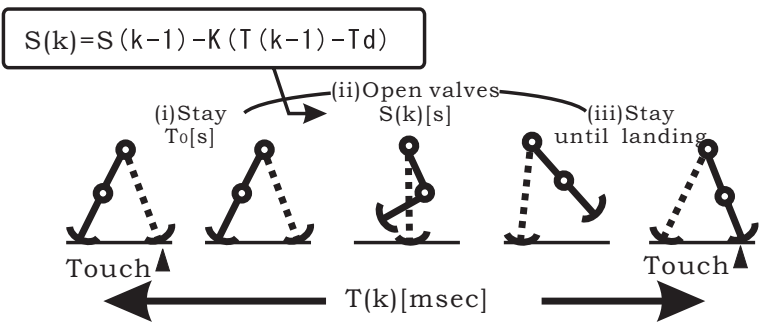

Fig. 4. Walking pattern and the controller

\section{Experimental results}

\subsection{Relationship between the duration of opening valves and walking cycle}

We observe the relationship between the input durations of opening valves to swing leg $S(k)$ and the output walking cycles $T(k)$.

In the experiment, the robot walks on the plane with the fixed duration of opening valves $S(k)=320[\mathrm{~ms}]$, and on the pre-determined step, the duration is changed randomly between $200[\mathrm{~ms}]$ to $440[\mathrm{~ms}]$. The result shown in Fig. 5 indicates the relationship between the duration of opening valves and walking cycle. The duration and the cycle have a positive correlation in each case. The correlation rate is 0.72 when the duration of opening valves to swing a pair of inner legs is changed. As a result of using a real robot, it is confirmed that the walking cycle is influenced by the duration of opening valves, and eqn.(1) is an effective law to control walking cycle.

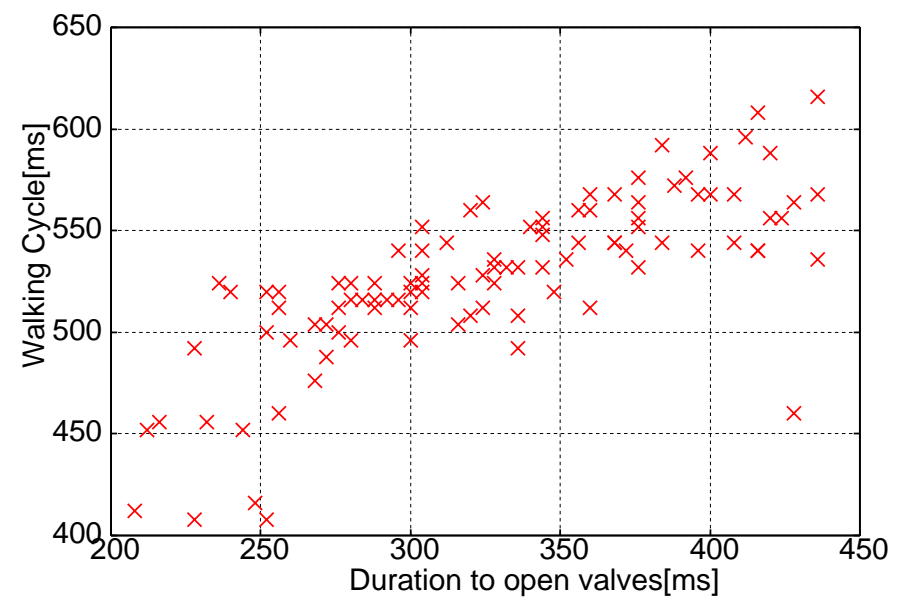

Fig. 5. Relation between the walking cycles and the durations of opening valves to rotate hip joint when a pair of inner legs swings. The cycle and the duration have positive correlation. 
The cycle of the steps is different when outer and inner legs swings because the dynamics of each pair of legs is different. In subsequent experiments, the desired walking cycle when the pair of outer and inner legs swings is set as $T_{d}=640[\mathrm{~ms}]$ and $T_{d}=560[\mathrm{~ms}]$, respectively.

\subsection{Testing negotiation ability with terrain changes}

\section{descending one difference in level}

In order to evaluate the proposed control, we have an experiment to walk over one small difference in level of $4[\mathrm{~mm}]$ using the biped walker.

Fig6 shows variations of the cycles (a)with / (b)without cycle control by 10 trials in each case. In each figure, the dots " $x$ " on lines express the walking cycles. The walker descends at 11th step, and the walking cycle becomes shorter in both case. As shown in Fig.6 (a), the walking cycle remains the same as before descending when the robot walks with the control. As shown in the Fig.6 (b), the robot often falls down at 15 th step if it walks without control. Sometimes the robot without controller walks over the difference, but the cycles are kept shorter and do not indicate fixed patterns.

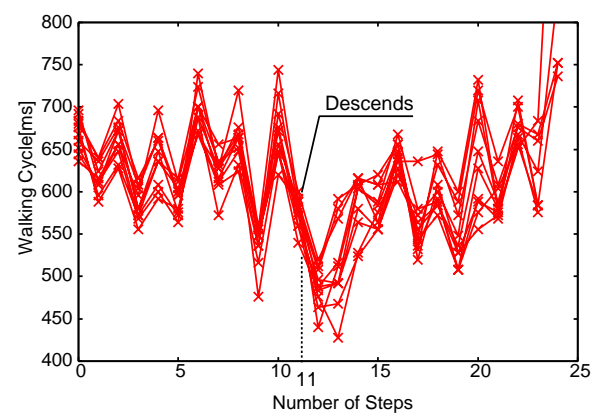

(a) With controller. After the 20th step, the cycle remains the same as before descending.

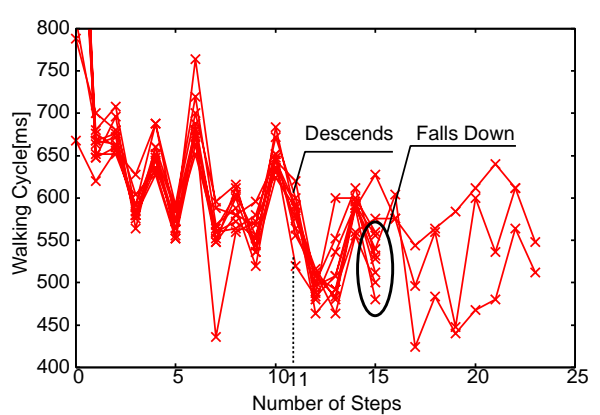

(b) Without controller. The robot often falls down at the 15 th step or continues to walk getting its cycle faster

Fig. 6. The result of walking with/without the control when the robot walks over the one differences in level

\section{descending two differences in level}

In the previous experiment, the walking cycle gets faster than before descending when the robot walks without control. We then have a experiment to walk over 
two differences in level shown in Fig.7. In results, the robot with control can walk over two differences as shown in the Fig.7 72 times of 100 trials, and the robot without control can walk over 27 times of 100 trials. The walking cycle of the robot without control gets more faster so that swing leg hits on the ground before swinging fully. The results of descending differences show that proposed control is effective to control walking cycle, and it can be said that the controller stabilize the walking against the terrain changes.

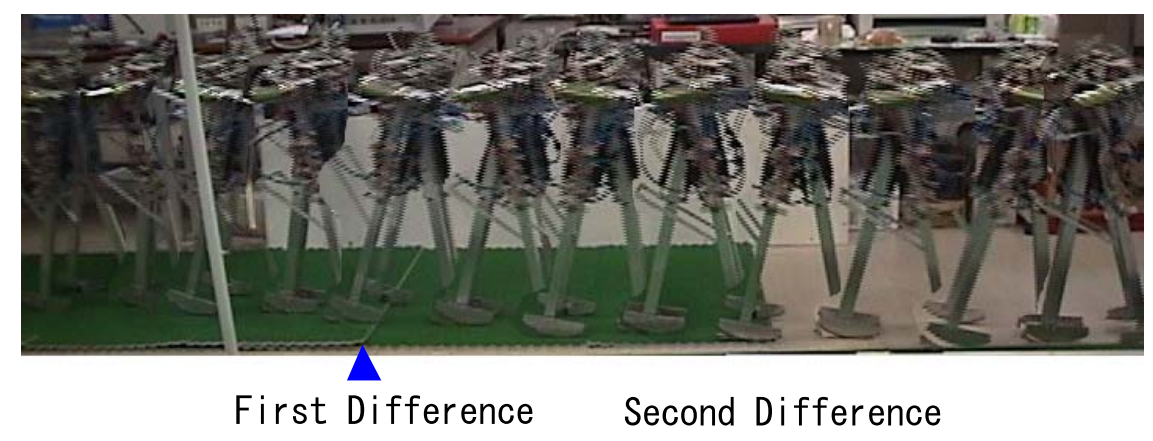

Fig. 7. The result of walking with the control when the robot walks over two differences in level

\section{Conclusion}

This paper introduces the developed biped walker that has McKibben artificial muscles. The height, width, and weight of the robot are $0.750[\mathrm{~m}], 0.350[\mathrm{~m}]$, and $5[\mathrm{~kg}]$, respectively. It has three degrees of freedoms, and it is designed to be self-contained.

Although the antagonistic pair of McKibben muscles is suitable for the quasipassive dynamic walking, it is difficult to control the actuator because of its complex nonlinearity. We design the robot to realize walking with simple operation of opening / closing the air valves that drive the actuators to make the locomotion. We propose a simple control law for stable walking by changing the duration to swing the leg.

In the experiments, we observed first the relation between a duration of opening valves and a walking cycle using the real robot. In results, the duration and the walking cycle had positive correlation, which means that the walking cycle could be controlled by changing the duration of opening valves. Then the simple control law is proposed and we let the robot walk over one difference in level with and without control. When the robot walked without control, the walking cycle was kept shorter after descending the difference, but the cycle with the control resumes to be as long as before descending the difference. The robot with control could often walk over two differences in level, but the robot without control could not so often walk over.

Our final goal is to build a robot that can walk against larger perturbation, and perform more dynamic motions such as running and jumping. In order to realize such motions, we are now planning to build up the mechanism of the robot which 
can change its dynamics easily, such as adding upper body or ankle joints. This is a future work.

\section{References}

1. T. McGeer, Passive dynamic walking, International Journal of Robotic Research, Vol.9, No.2, pp.62-82, 1990

2. M. Wisse and J. van Frankenhuyzen, Design and Construction of MIKE; 2D autonomous biped based on passive dynamic walking, 2nd International Symposium on Adaptive Motion of Animals and Machines, WeP-I-1, October, 2002

3. Y.Sugimoto, K.Osuka, Walking Control of Quansi-Passive-Dynamics-Walking Robot "Quartet III" based on Delayed Feedback Control, Proceedings of 5th International Conference on Climbing and Walking Robots (CLAWAR 2002), Paris, France, 2002, September.

4. Fumihiko Asano, Masaki Yamakita, and Katsuhisa Furuta Virtual Passive Dynamic Walking and Energy-based Control Laws, Proceedings of the 2000 IEEE/RSJ International Conference on Intelligent Robots and Systems, pp.1149-1154, 2000

5. Fumihiko Asano, Masaki Yamakita, Norihiko Kawamichi, and Zhi-Wei LUO, $A$ Novel Gait Generation for Biped Walking Robots Based on Mechanical Energy Constraint, Proceedings of the 2002 IEEE/RSJ Intl. Conference on Intelligent Robots and Systems EPFL, Lausanne, Switzerland, 2002, October

6. Richard Q. van der Linde, Design, Analysis, and Control of a Low Power Joint for Walking Robots, by Phasic Activation of McKibben Muscles, IEEE Transactions on Robotics and Automation, Vol.15, No.4, August 1999

7. Darwin G. Caldwell, Gustavo A. Medrano-Cerda, and Mike Goodwin, Control of pneumatic muscle actuators, IEEE Control Systems, pp.40-47, February 1995

8. Joachim Schröder, Kazuhiko Kawamura, Tilo Gockel, and Rüdiger Dillmann, Improved control of humanoid arm driven by pneumatic actuators, International Conference on Humanoid Robots 2003, CD-ROM, 2003

9. R. V. Ham, B. Verrelst, F. Daerden, and D. Lefeber, Pressure control with on-off valves of Pleated Pneumatic Artificial Muscles in a modular one-dimensional rotational joint, International Conference on Humanoid Robots, CD-ROM, October 2003

10. HITACHI Medical corp. web site : http://www.hitachi-medical.co.jp/english/index.htm

11. SMC Corporation web site : http://www.smcworld.com/ 